\title{
CtBPI Mediates Hypoxia-Induced Sarcomatoid Transformation in Hepatocellular Carcinoma
}

\author{
Xiaoling Zhang $\mathbb{D}^{1,2, *}$, Xiaoyu Wang ${ }^{3, *}$, Liting Jia ${ }^{4}$, Yang Yang ${ }^{5}$, Fan Yang ${ }^{3}$, Shengjun Xiao $\mathbb{D}^{2,3,6}$ \\ 'Department of Physiology, Faculty of Basic Medical Science, Guilin Medical University, Guilin, People's Republic of China; ${ }^{2}$ Key Laboratory of Tumor \\ Immunology and Microenvironmental Regulation of Guangxi, Guilin Medical University, Guilin, People's Republic of China; ${ }^{3}$ Department of Pathology, \\ the Second Affiliated Hospital of Guilin Medical University, Guilin, People's Republic of China; ${ }^{4}$ Department of Pathology, Affiliated Hospital of Hebei \\ Engineering University, Handan, People's Republic of China; ${ }^{5}$ Department of Pathology, General Hospital of Central Theater Command, Wuhan, \\ People's Republic of China; ${ }^{6}$ Department of Pathology, Guangxi Health Commission Key Laboratory of Glucose and Lipid Metabolism Disorders, the \\ Second Affiliated Hospital of Guilin Medical University, Guilin, People's Republic of China
}

*These authors contributed equally to this work

Correspondence: Shengjun Xiao, Department of Pathology, The Second Affiliated Hospital, Guilin Medical University, No. 2I2, Renmin Road, Guilin, Guangxi Province, 54I 199, People's Republic of China, Email xiaoshengjun@glmc.edu.cn; Xiaoling Zhang, Department of Physiology, Faculty of Basic Medical Science, Guilin Medical University, No. I, Zhiyuan Road, Guilin, Guangxi Province, 54I I00, People's Republic of China, Email rose2006@glmc.edu.cn

Background: Sarcomatoid hepatocellular carcinoma (sHCC), a highly aggressive subtype of hepatocellular carcinoma (HCC), mostly transforms from classical hepatocellular carcinoma (cHCC). The study intended to explore the role of C-terminal binding protein 1 (CtBP1) in sarcomatoid transformation of hepatocellular carcinoma.

Methods: Western blotting and/or immunohistochemistry were used to confirm the expression of CtBP1 and other proteins in HCC cells, xenografts and clinical tissue samples. CtBP1 shRNA-expressing lentivirus was used to infect HepG2 cells to construct CtBP1 knockdown cells. Cell migration was determined by scratch wound assays and Transwell assays. Immunofluorescence was used to label the a-tubulin cytoskeleton to evaluate cell morphology. HepG2 cells were inoculated subcutaneously in nude mice to construct xenografts and beneath the liver capsule to evaluate in vivo metastasis.

Results: Compared to that in the $\mathrm{cHCC}$ area, $\mathrm{CtBP} 1$ expression was significantly upregulated in the sHCC area, as shown by immunohistochemistry. HE staining showed that cells in the $\mathrm{sHCC}$ area were spindle-shaped, while those in the $\mathrm{cHCC}$ area were polygonal. Immunohistochemically, the epithelial markers pancytokeratin (CK) and E-cadherin were partially or completely lost, while the expression of the mesenchymal marker vimentin was upregulated in the sHCC area. Moreover, HepG2, an HCC cell line with high expression of CtBP1, autonomously underwent sarcomatoid transformation, showing a sarcomatoid morphology and phenotype. HIF1a expression was upregulated in epithelial cells adjacent to the sHCC area. Hypoxia upregulated CtBP1 protein expression and induced an EMT phenotype with increased migration and a spindle-shaped morphology in HepG2 cells. Knockdown of CtBP1 partially reversed the EMT phenotype induced by hypoxia. Silencing CtBP1 completely blocked the sarcomatoid transformation of subcutaneous xenografts and decreased lung metastasis in subcapsular xenografts of the liver in nude mice.

Conclusion: CtBP1 plays a key role in hypoxia-induced EMT and sarcomatoid transformation in HCC and could be a candidate target for the management of sHCC.

Keywords: C-terminal binding protein 1, sarcomatoid hepatocellular carcinoma, hypoxia, sarcomatoid transformation, epithelial-tomesenchymal transition

\section{Introduction}

Sarcomatoid hepatocellular carcinoma (sHCC), a histological variant of classical hepatocellular carcinoma (HCC), is partially or entirely composed of malignant spindle cells. ${ }^{1}$ The incidence of sHCC ranges from $1.8 \%$ to $3.9 \%$ of all $\mathrm{HCC}$ cases. $^{2,3}$ sHCC can be the primary tumor but is mainly secondary tumors in patients undergoing repeated chemotherapy, transarterial chemoembolization (TACE), radiofrequency ablation (RFA), and percutaneous ethanol injection. ${ }^{1}$ sHCC shows a more aggressive phenotype with a higher TNM stage and worse prognosis than classic HCC (cHCC). ${ }^{4}$ ecently,

Received: 28 September 2021 Accepted: 2 February 2022

Published: 11 February 2022 


\section{Graphical Abstract}

\section{Hypoxia induced sarcomatoid transformation}

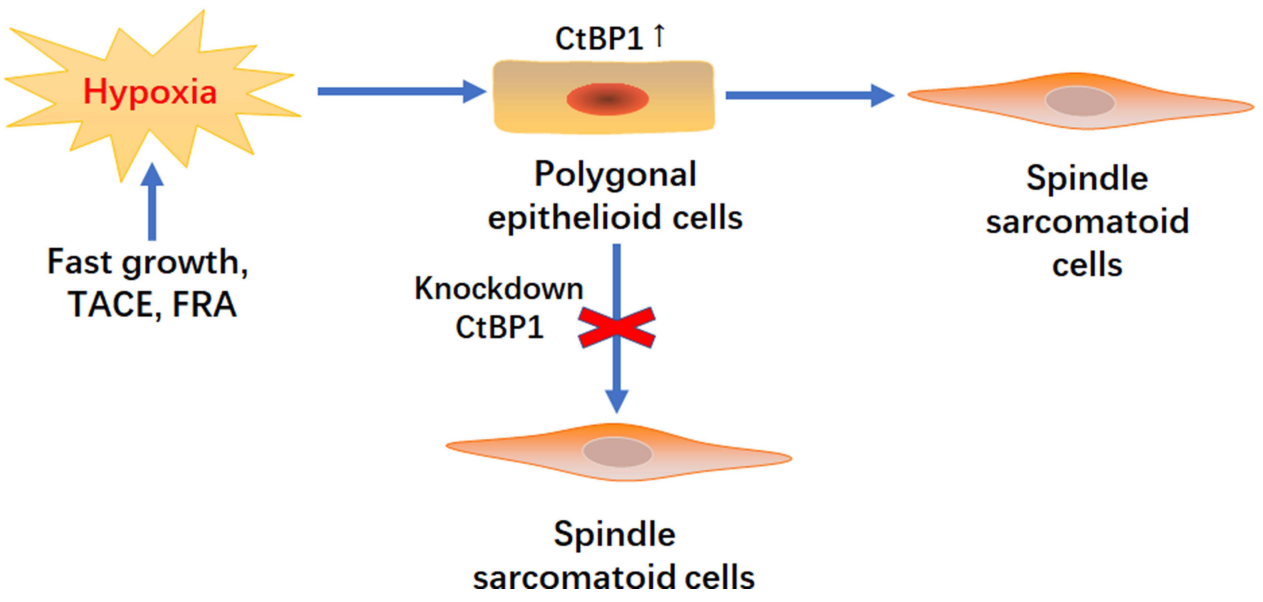

distinct immunomicroenvironmental characteristics with upregulation of the expression of multiple immune checkpoint molecules, which suggest promising prospects in immunotherapy in sHCC, have been reported. ${ }^{5}$

Although some candidate oncogenic drivers have been reported, ${ }^{6}$ the molecular basis of sarcomatoid transformation in HCC is still largely unknown. Sarcomatoid transformation of cancer is considered a classic model of epithelial-tomesenchymal transition (EMT) of cancer progression. ${ }^{7,8}$ Hypoxia is thought to be an initiator of sarcomatoid transformation during tumor progression. ${ }^{9} \mathrm{C}$-terminal binding protein 1 (CtBP1), an oxygen sensor of the tissue microenvironment and a transcriptional corepressor of E-cadherin, was reported to play a key role in hypoxia-induced EMT of cancer cells. ${ }^{10}$ Previously, we reported that CtBP1 promoted EMT in HCC cells. ${ }^{11}$ In this study, we report that CtBP1 plays an essential role in hypoxia-induced sarcomatoid transformation of HCC.

\section{Methods}

\section{Tissue Samples and Cell Culture}

Thirteen human paraffin-embedded biopsies of sarcomatoid hepatocellular carcinoma tissues were obtained from the Department of Pathology, Second Affiliated Hospital of Guilin Medical College (Guilin, China) from 2008 to 2015. The clinicopathological diagnosis of all biopsied tissues was primary sarcomatoid hepatocellular carcinoma, and none of the patients had received surgery or other radiotherapy prior to surgical resection. The utilization of human materials was approved by the Biomedical Ethics Committee of the Second Affiliated Hospital of Guilin Medical College (No. GLMC201710920) and in accordance with the Declaration of Helsinki. HepG2 cells, previously purchased from ATCC (Cat. No. HB-8065 TM), were maintained in Prof. Xiaoling Zhang's laboratory at the School of Basic Medicine, Guilin Medical College (Guilin, China) and authenticated by STR profiling. HepG2 cells were cultured in Dulbecco's modified Eagle's medium (DMEM) (Gibco, Grand Island, NY, USA) supplemented with $10 \%$ fetal bovine serum. Control $\left(21 \% \mathrm{O}_{2}\right)$ cells were maintained at $37^{\circ} \mathrm{C}, 5 \% \mathrm{CO}_{2}$, and $95 \%$ relative humidity in a cell incubator; cells in the hypoxic culture group $\left(1 \% \mathrm{O}_{2}\right)$ were maintained at $37^{\circ} \mathrm{C}, 5 \% \mathrm{CO}_{2}$, and $95 \%$ relative humidity in an anaerobic cell incubator.

\section{In vivo Tumorigenesis Assay}

Animal experiments were approved by the Animal Research Committee of Guilin Medical College (No. EFY-GZR008). All animal experiments were performed in accordance with the National Institutes of Health Guidelines for the Care and 
Use of Laboratory Animals. All nude mice in this study were maintained in microisolator cages under aseptic conditions. HepG2 stable cells $\left(5 \times 10^{6}\right)$ were resuspended in $200 \mu \mathrm{L}$ of PBS and injected subcutaneously into the right forelimb axilla of 4-week-old thymus-free BALB/C nude mice purchased from the Experimental Animal Center of Guilin Medical College. Nude mice were sacrificed after 4 weeks, and the transplanted tumors were isolated, photographed and formalin fixed.

\section{In vivo Metastasis Assay of Nude Mice}

Sodium pentobarbital sulfate $(0.6 \% ; 40-50 \mathrm{mg} / \mathrm{kg})$ was injected intraperitoneally to anesthetize 4-week-old thymus-free $\mathrm{BALB} / \mathrm{C}$ nude mice, and the abdomen was opened. Then, control or shCtBP1-expressing HepG2 cells $\left(5 \times 10^{6}\right)$ resuspended in $100 \mu \mathrm{L}$ of PBS were injected beneath the capsule of the liver. Finally, the liver was retracted into the abdominal cavity, and the abdomen was closed with interrupted sutures. Nude mice were sacrificed after 5 weeks, and the lung metastases were counted and photographed.

\section{Hematoxylin-Eosin (HE) Staining and Immunohistochemistry (IHC)}

For HE staining, after dewaxing and dehydration, 3- $\mu \mathrm{m}$-thick tissue sections were stained with hematoxylin-eosin for 3 min each, fractionated with hydrochloric acid ethanol, and sealed with neutral gum.

For immunohistochemical staining, tissue blocks were sectioned into 3- $\mu \mathrm{m}$-thick sections. After dewaxing and rehydration, high temperature-induced epitope retrieval (HIER) was conducted in a high-pressure cooker for 3 minutes in citrate buffer $(0.01 \mathrm{M}, \mathrm{pH} 6.0)$. Endogenous hyperoxidase was blocked with $3 \% \mathrm{H}_{2} \mathrm{O}_{2}$ for 10 minutes, and then, primary antibodies (information on antibodies is summarized in Table S2) were incubated overnight at $4{ }^{\circ} \mathrm{C}$. Then, slides were incubated with secondary antibodies for 30 minutes at room temperature. Furthermore, 3,3'-diaminobenzidine (DAB) was used as a chromogen, and the nuclei were counterstained with hematoxylin.

The semiquantitative score was determined by two experienced pathologists with a double-blind method, and the criteria were briefly as follows: 1) Staining intensity score: 0 points for no color, 1 point for faint yellow, 2 points for yellow, and 3 points for brown. 2) Positive cell percentage score: $<10 \% 0$ points, $10-30 \% 1$ point, 30-50\% 2 points, $>50 \% 3$ points. Total score $=$ staining intensity score $\times$ positive cell percentage score. A score of 0 was defined as negative (-), 1-4 as weakly positive (+), 5-6 as moderately positive (++), and $\geq 6$ as strongly positive (+++). Finally, a score of $\leq 4$ was defined as low expression, and a score of 5-9 was defined as high expression.

\section{Construction of Lentivirus-Infected Cells}

Lentiviruses expressing shCtBP1 and blank control shRNA (shSCR) were purchased from Abcam. According to the manufacturer's instructions, $4 \times 10^{4}$ cells per well were inoculated with lentivirus for transfection. Infected cells were screened with $5 \mu \mathrm{g} / \mathrm{mL}$ puromycin (Solarbio life Science, Beijing, China) for more than 1 week, and transfection efficiency was detected by Western blots.

\section{Western Blot Assays}

Whole-cell lysates were prepared to extract the total protein, which was separated by $10 \%$ PAGE and transferred to a polyvinylidene fluoride membrane. The membrane was blocked with $5 \%$ nonfat milk at room temperature for $1 \mathrm{~h}$, followed by incubation with the primary antibody at $4{ }^{\circ} \mathrm{C}$ overnight and incubation with the horseradish peroxidase (HRP)-conjugated secondary antibody at room temperature for 1.5 hours. Finally, the chemiluminescence (ECL) signal was detected using ECL reagent (FDbio Science, Hangzhou, China). Information on the primary antibodies and secondary antibodies used in this study is summarized in Table S2.

\section{Immunofluorescence Assay}

Briefly, $8 \times 10^{3}$ HepG2 cells were inoculated in crawl sheets, fixed with $4 \%$ paraformaldehyde (Solarbio), permeabilized with $0.5 \%$ Triton X-100 (Solarbio), and blocked with $10 \%$ fetal bovine serum at room temperature for 30 minutes. The fluorescent primary antibody was incubated at room temperature for 2 hours, PBST was used for 3 washes, the 
fluorescent secondary antibody was incubated at $37{ }^{\circ} \mathrm{C}$ for 1 hour, and the nuclei were counterstained with DAPI. The fluorescent antibodies used in this study are summarized in Table S2.

\section{Transwell Migration Assays}

Cell migration was observed using Transwell chambers (Corning). Cells were collected and resuspended in serum-free medium, $200 \mu \mathrm{L}$ of cell suspension at a density of $5 \times 10^{5}$ cells per $\mathrm{mL}$ was placed in the upper chamber, and $600 \mu \mathrm{L}$ of DMEM high sugar medium containing $10 \%$ fetal bovine serum was added to the lower chamber. After 24-48 hours of incubation, cells were fixed with $4 \%$ paraformaldehyde (Solarbio) for 20 minutes at $4{ }^{\circ} \mathrm{C}$ and then stained with $0.1 \%$ crystal violet (Sigma) for 30 minutes.

A scratch healing assay was also employed to observe cell migration. Monolayers of cells were equally scratched with a narrow edge of a $10 \mu \mathrm{L}$ pipette tip and cultured in serum-free DMEM medium. The healing distance of the scratched wounds was then recorded under a $20 \times$ light microscope at 24 and 48 hours.

\section{Statistical Analysis}

SPSS 23.0 (SPSS, Inc.) was used for statistical analysis, and GraphPad Prism 6.0 software (GraphPad, Inc.) was employed to draw statistical graphs. The results are shown as the mean $\pm \mathrm{SD}$. One-way ANOVA and paired $t$-tests were used to compare protein levels, mobility and other measures between different groups. The level of statistical significance was defined as $\mathrm{P}<0.05(* \mathrm{P}<0.05 ; * * \mathrm{P}<0.01 ; * * * \mathrm{P}<0.001)$.

\section{Results}

\section{CtBPI Expression Was Upregulated in Sarcomatoid HCC Tissues, and Xenografts of HepG2 Cells Autonomously Underwent Sarcomatoid Transformation}

Sarcomatoid hepatocellular carcinoma (sHCC), a histological variant of classical hepatocellular carcinoma (cHCC), often shows sarcomatoid morphology and an immunophenotype with epithelial-to-mesenchymal transition. Histologically, the differential diagnosis of sarcoma and sarcomatoid hepatocellular carcinoma is difficult. The remaining untransformed cHCC area is an important clue for making a correct diagnosis. As shown in Figure 1A and Table S1, HE staining showed that cancer cells in $\mathrm{cHCC}$ areas were polygonal and cancer cells in sHCC areas were spindle-shaped. Immunohistochemically, the levels of the epithelial markers CK and E-cadherin were significantly downregulated and the expression of the mesenchymal marker vimentin was significantly increased in the sarcoma-like region compared with the epithelial area. The above morphology and immunophenotype suggested that sarcomatoid transformation of hepatocellular carcinoma is a paradigm of EMT. In addition, upregulated CtBP1 expression indicated its potential role in sarcomatoid transformation. HepG2 cells, an HCC cell line with high CtBP1 expression, autonomously underwent sarcomatoid transformation. As shown in Figure 1B, at the center of HepG2 cell xenografts, tumors showed necrosis, and cells at the periphery of the necrotic area showed a spindle-shaped morphology adjacent to the epithelial area. Moreover, the levels of the epithelial markers CK and E-cadherin were downregulated, and the expression of the mesenchymal marker vimentin was upregulated (Figure 1C). Morphology and immunophenotype data suggest EMT or sarcomatoid transformation in HepG2 xenografts. It has been reported that hypoxia frequently presents in the center of tumors and promotes progression. ${ }^{12}$ In this study, HIF $1 \alpha$ expression was upregulated in epithelioid cancer cells adjacent to sarcomatoid areas, suggesting a hypoxic microenvironment near sarcomatoid areas (Figure 1D). However, interestingly, the sarcomatoid area lost the expression of HIF1 $\alpha$ (Figure 1D), which suggests that hypoxia do not modulate the expression of CtBP1 through HIF1a and hypoxia is not necessary for maintaining the sarcomatoid state once sarcomatoid transformation is finished. HIF $1 \alpha$ may be an early initiating factor driving sarcomatoid transformation. These results were consistent with the data reported in clinical tissue samples of renal cell carcinoma with sarcomatoid transformation, in which HIF $1 / 2 \alpha$ upregulated in epithelia area adjacent to sarcomatoid area while downregulated in sarcomatoid area. ${ }^{13}$ Therefore, CtBP1 might be involved in sarcomatoid transformation, and hypoxia was probably the driving factor of sarcomatoid transformation in HCC. 


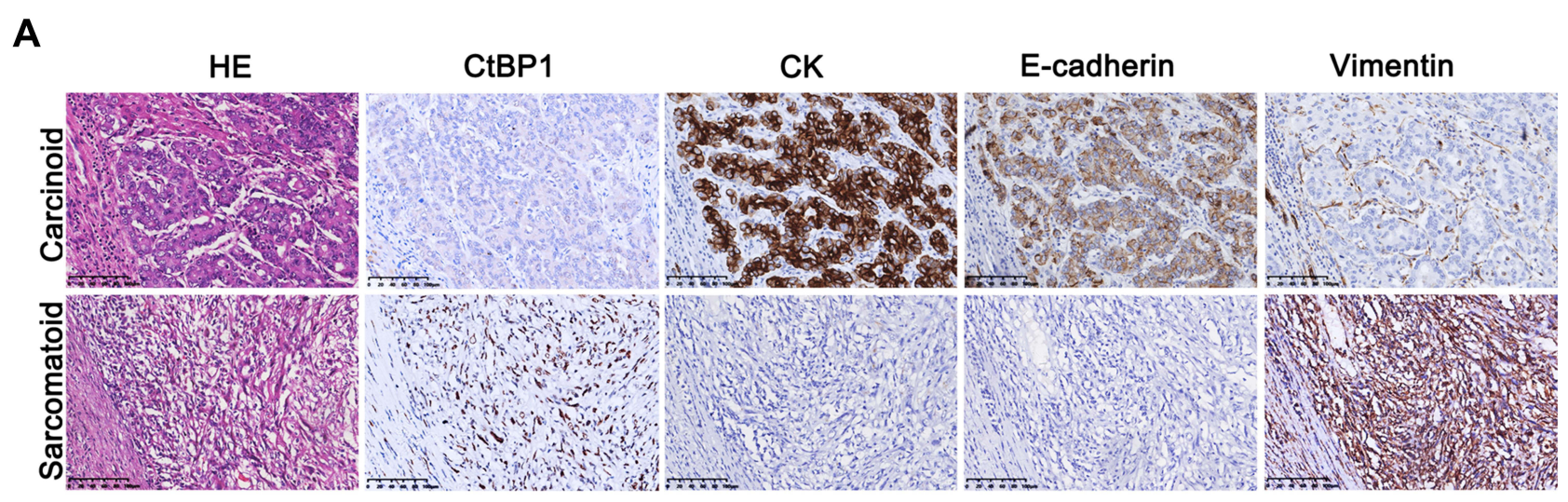

B

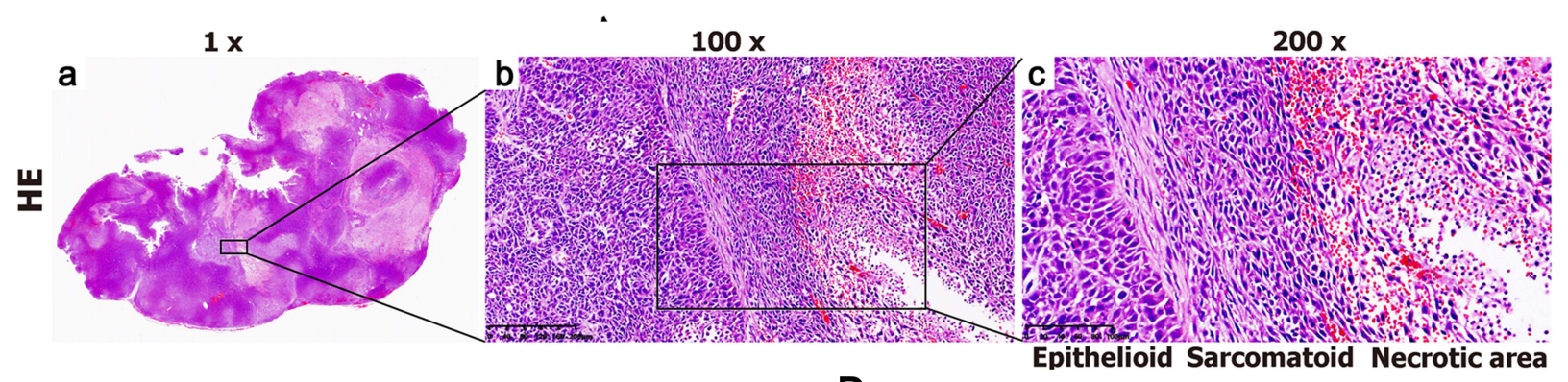

C
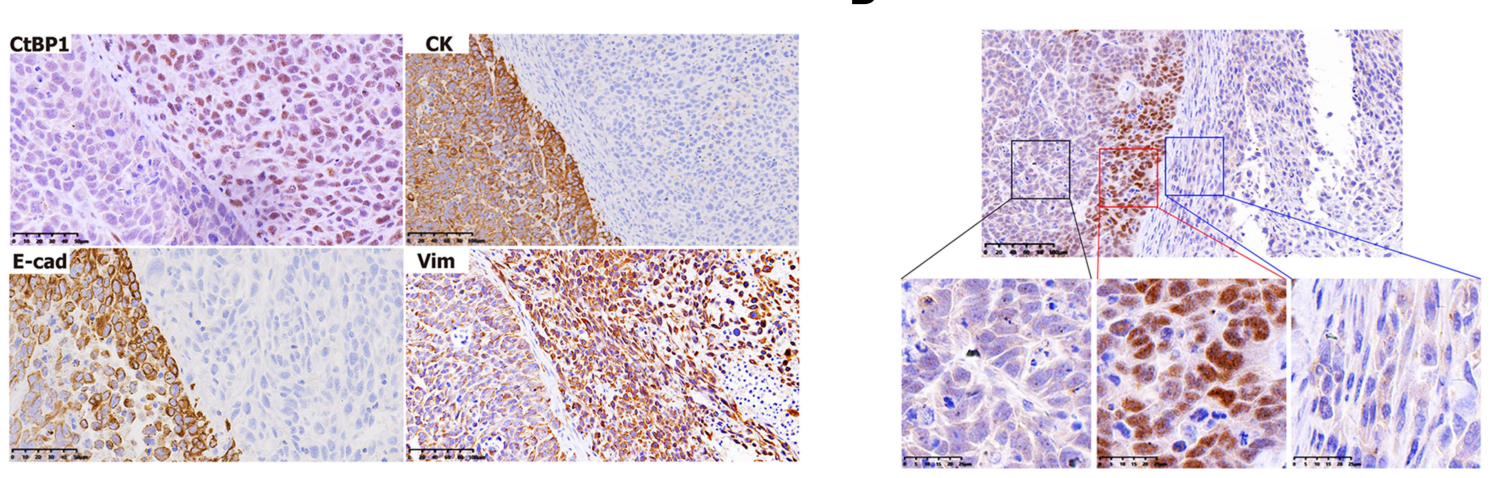

Figure I CtBPI expression is upregulated in sarcomatoid hepatocellular carcinoma tissues, and xenografts of HepG2 cells autonomously undergo sarcomatoid transformation. (A) In sarcomatoid hepatocellular carcinoma, cells in the sarcomatoid area are spindle-shaped, while those in the epithelioid area are polygonal-shaped (H \& E staining). Compared with those in the epithelioid area, CtBPI expression was upregulated, CK and E-cadherin expression was downregulated, and vimentin expression was upregulated in the sarcomatoid area (immunohistochemistry). (B) Adjacent to the necrotic area, HepG2 cells undergo sarcomatoid transformation in xenografts of nude mice. (C) Immunohistochemically, CtBPI expression was upregulated, CK and E-cadherin expression was downregulated, and vimentin expression was upregulated in the sarcomatoid-transformed area in HepG2 xenografts. (D) HIF-I $\alpha$ expression was upregulated in the epithelioid area adjacent to the sarcomatoidtransformed area but not in other areas.

\section{Hypoxia Upregulated CtBPI Expression and Induced an EMT Phenotype in HepG2 Cells}

To elucidate the role of CtBP1 in hypoxia-induced sarcomatoid transformation, we cultured HepG2 cells, which showed high CtBP1 expression, under hypoxic conditions. Western blot analysis showed that CtBP1 expression was continuously upregulated by hypoxia at 6,12 , and 24 hours. Moreover, the expression of the epithelial adhesion protein E-cadherin was persistently downregulated in HepG2 cells (Figure 2A). The expression of the oxygen-sensing factor HIF-1 $\alpha$ was upregulated by hypoxia at 6,12 and 24 hours but downregulated at 24 hours compared with that at 6 and 12 hours. Furthermore, hypoxia promoted cell migration at 24 and 48 hours (Figure 2B and C). Immunofluorescence assays showed that the cell morphology transformed from an epithelial-like polygonal shape to a mesenchymal-like spindle shape with downregulated expression of the epithelial marker E-cadherin and upregulated expression of the 
A

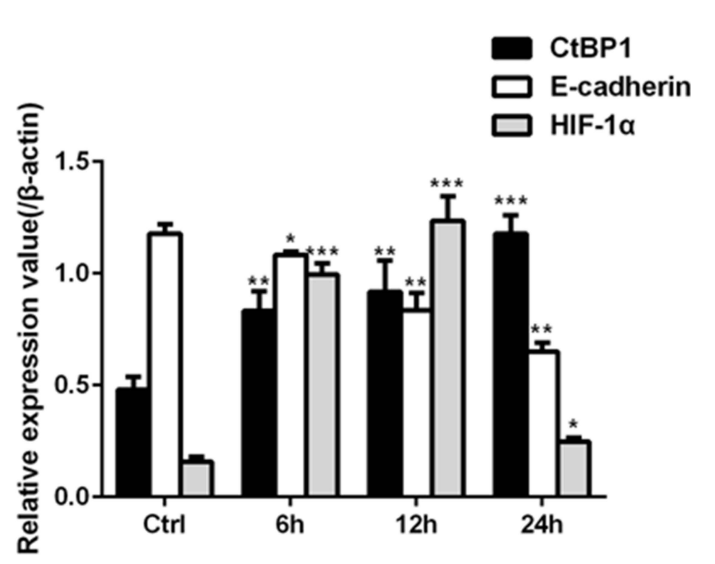

B
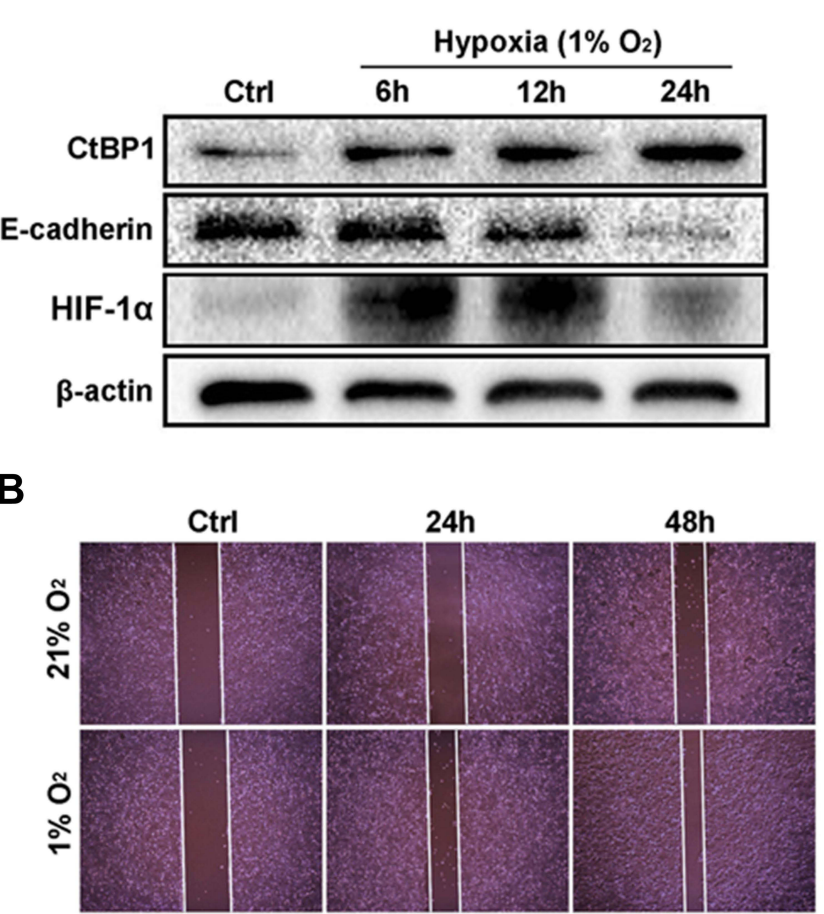

C

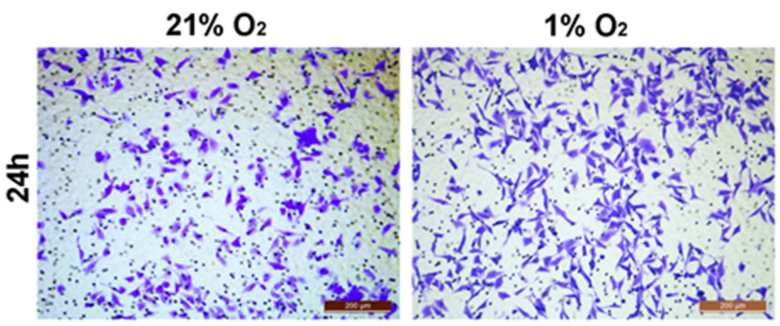

D

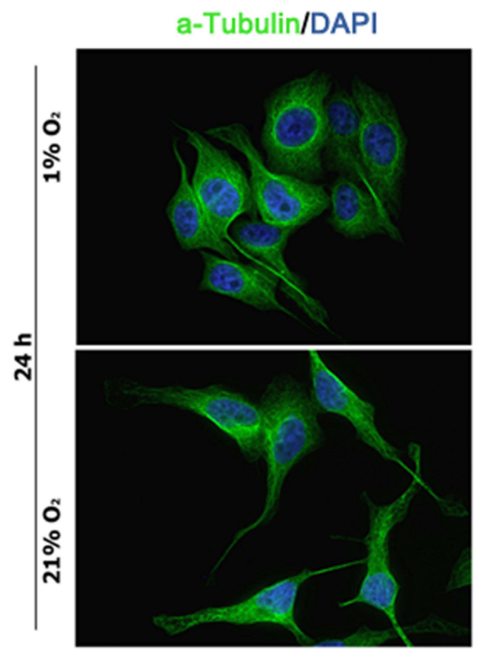

E-cad/CtBP1/DAPI
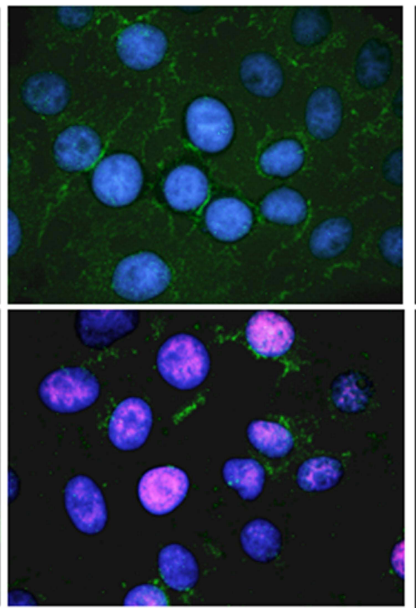
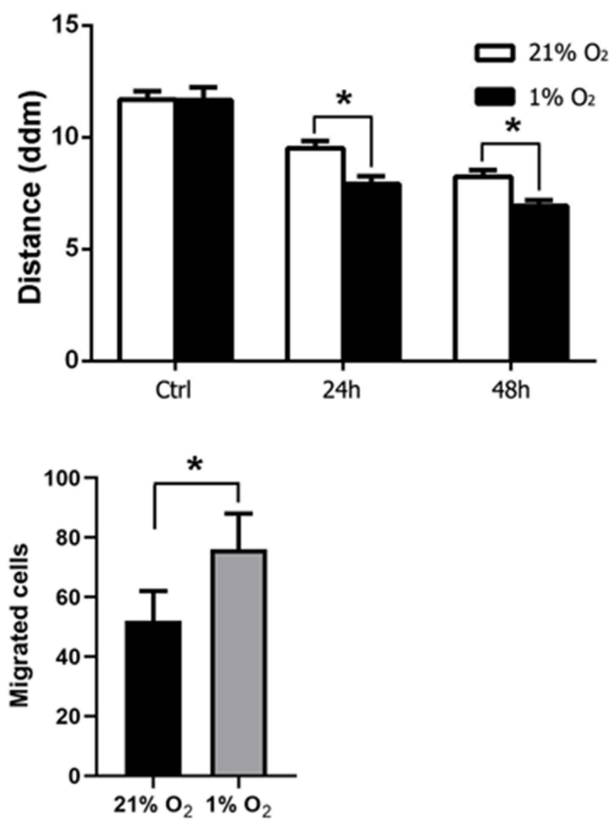

Vim/CtBP1/DAPI

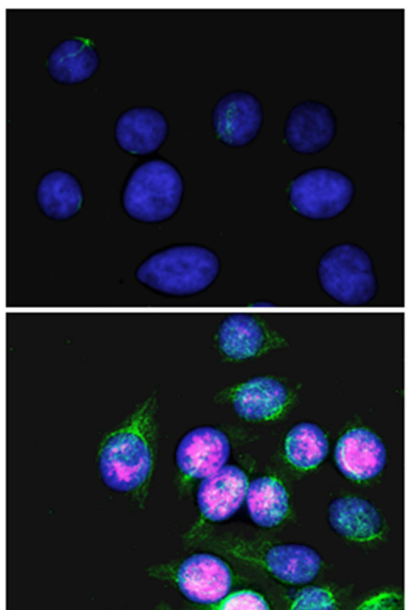

Figure 2 Hypoxia upregulates CtBPI expression and induces epithelial-to-mesenchymal transition (EMT) in HepG2 cells. (A) Hypoxia (I\% $\left.\mathrm{O}_{2}\right)$ upregulated CtBPI protein expression and downregulated E-cadherin expression at 6, 12, and 24 hours in HepG2 cells. HIF-I $\alpha$ expression was upregulated at 6, 12 , and 24 hours, while the increase in HIF-I $\alpha$ expression was decreased at 24 hours. (B) Wound scratch assays and (C) Transwell assays showed that hypoxia increased the mobility of HepG2 cells at 24 and/or 48 hours. (D) Immunostained $\alpha$-tubulin shows that hypoxia induces a spindle-shaped morphology in HepG2 cells with downregulated E-cadherin expression and upregulated vimentin expression. $* \mathrm{P}<0.05 ; * \mathrm{P}<0.01$; $* * * \mathrm{P}<0.001$. Compare with the control $(\mathrm{Ctrl})$ in Figure $2 \mathrm{~A}$. 
mesenchymal marker vimentin (Figure 2D). These data indicated that hypoxia induced EMT and upregulated the expression of CtBP1 in HCC cells.

\section{Knockdown of CtBPI Reversed the Hypoxia-Induced EMT Phenotype in HepG2 Cells}

To confirm that CtBP1 mediated hypoxia-induced sarcomatoid transformation in HepG2 cells, we generated cell lines stably transfected with CtBP1 (Figure 3A). After successful knockdown of CtBP1, the expression of E-cadherin proteins, which was downregulated by hypoxia, was partially restored. Moreover, the increased migratory ability, which was induced by hypoxia, was partially reversed by CtBP1 knockdown (Figure 3B and C). In addition, the spindle-shaped morphology of HepG2 cells, which was induced by hypoxia, was partially reversed to an epithelioid polygonal shape (Figure 3D). Furthermore, hypoxia-induced downregulation of E-cadherin expression and upregulation of vimentin expression were partially reversed by CtBP1 silencing. These data indicated that CtBP1 mediated hypoxia-induced changes in HCC cells.

A
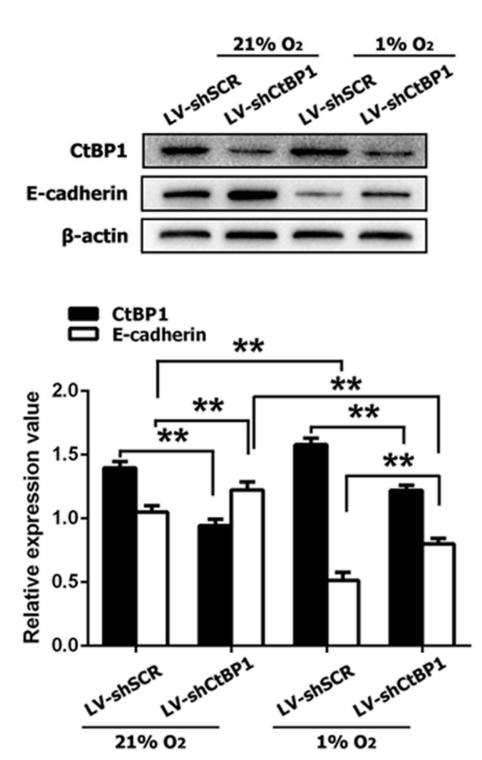

D
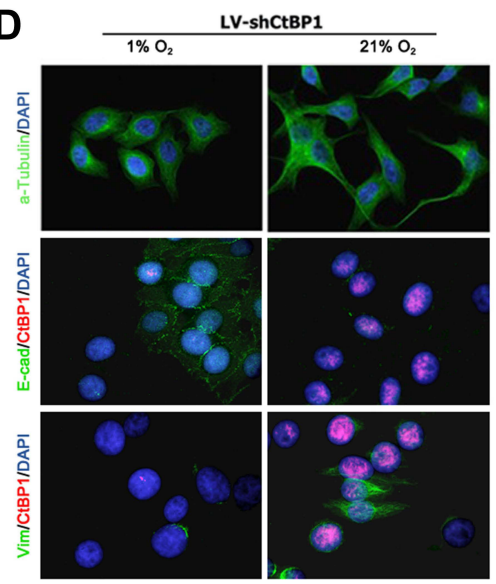

B
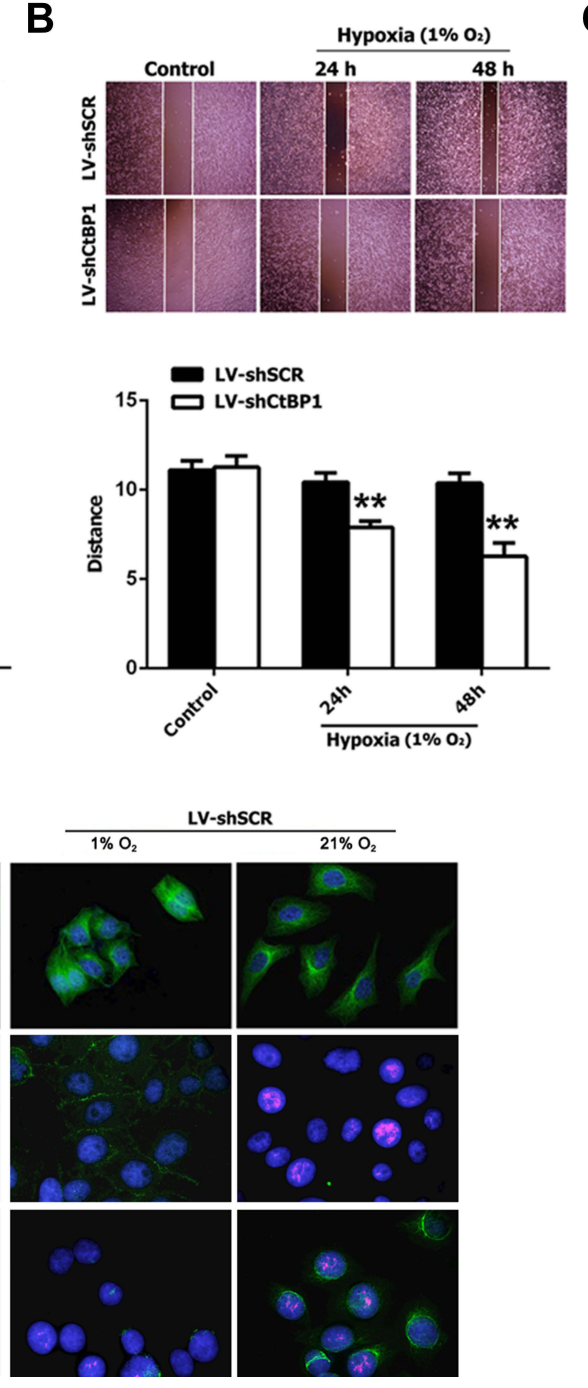

C
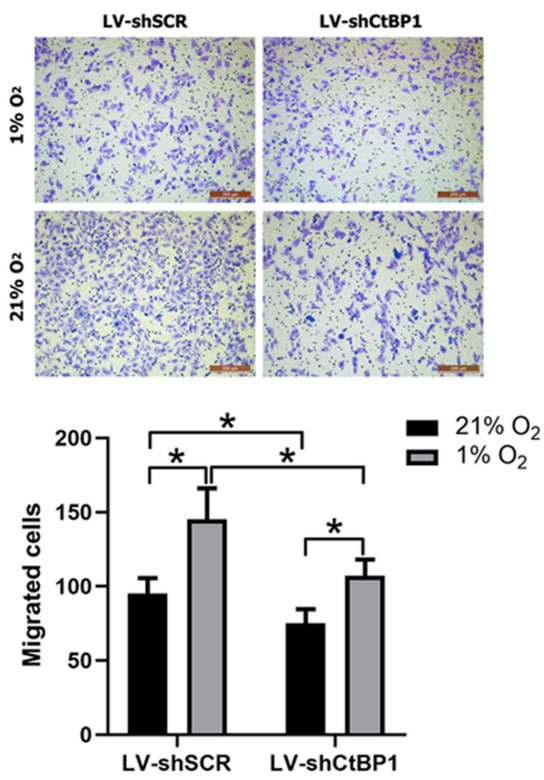

Figure 3 Knockdown of CtBPI reverses the hypoxia-induced EMT phenotype in HepG2 cells. (A) Knockdown of CtBPI partially rescued hypoxia-induced upregulation of $\mathrm{CtBPI}$ expression and downregulation of E-cadherin expression at 12 hours in HepG2 cells. (B) Wound scratch assays and (C) Transwell assays showed that silencing CtBPI inhibits the increased mobility stimulated by hypoxia in HepG2 cells at 24 and/or 48 hours. (D) Immunostained $\alpha$-tubulin shows that hypoxia-induced spindle-shaped morphology is reversed by CtBPI silencing in HepG2 cells. Moreover, hypoxia-induced downregulation of E-cadherin expression and upregulation of vimentin expression were reversed by $\mathrm{CtBPI}$ silencing. $* P<0.05 ; * P<0.01$. Compare with the control (Ctrl) in Figure 3B. 


\section{Knockdown of CtBPI Also Reversed Hypoxia-Induced Sarcomatoid Transformation in HepG2 Xenografts}

To identify hypoxia-induced changes in vivo, we subcutaneously inoculated HepG2 cells into nude mice to generate xenografts. The CtBP1 knockdown cells showed smaller tumor sizes and less necrosis in the center of the tumor than the control cells (Figure 4A and B). HE staining showed that the cancer cells adjacent to the necrotic area had a spindleshaped morphology in the control group, while tumor cells in the CtBP1 knockdown group maintained polygonal epithelial morphology (Figure 4B). Immunohistochemistry showed that most of the tumors lost the expression of the

A

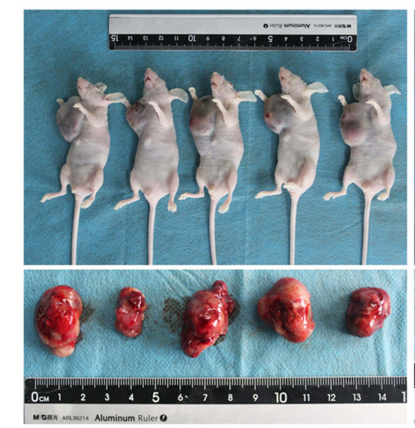

C

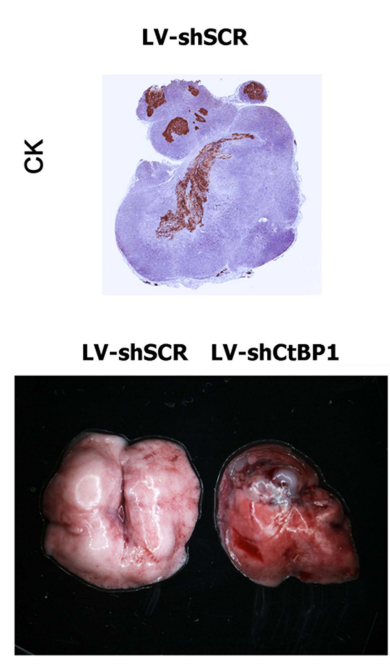

$\mathbf{F}$

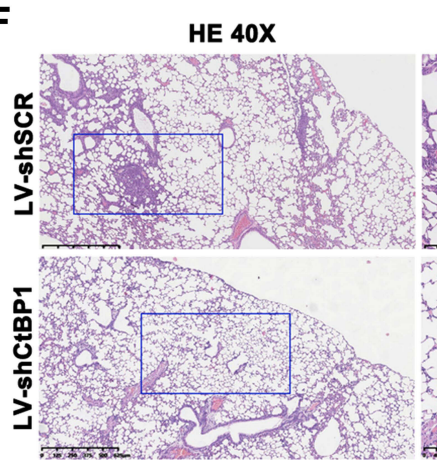

LV-shCtBP1

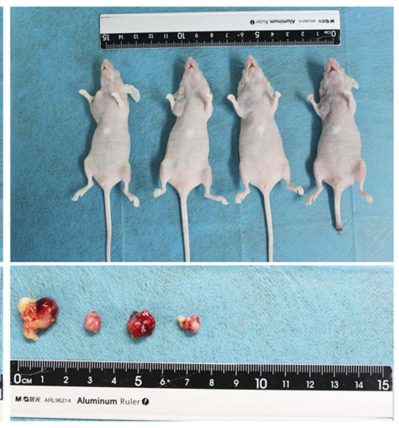

LV-shCtBP1

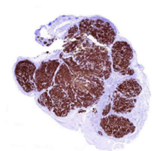

LV-shSCR LV-shCtBP1

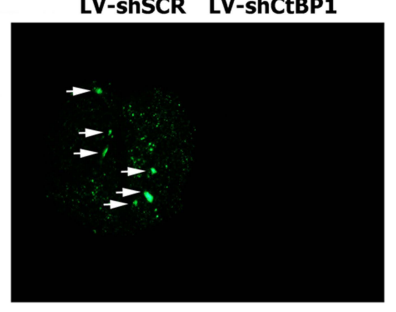

HE 100X

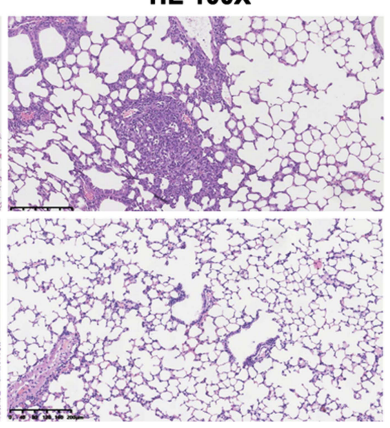

B
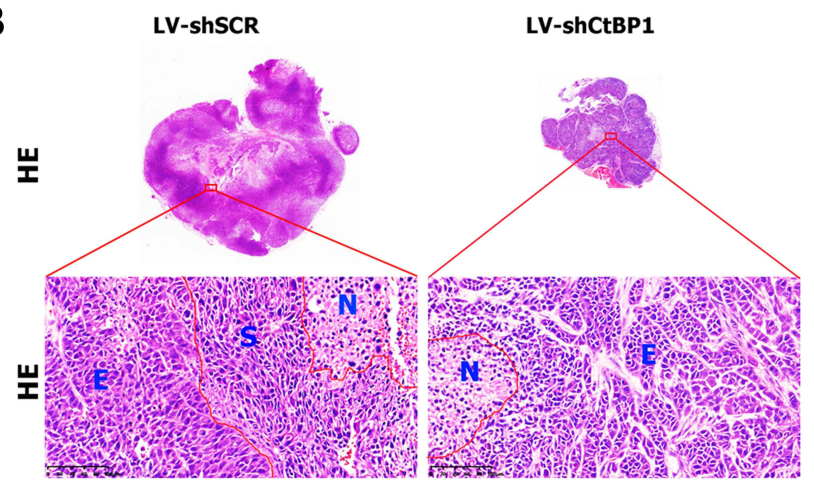

D

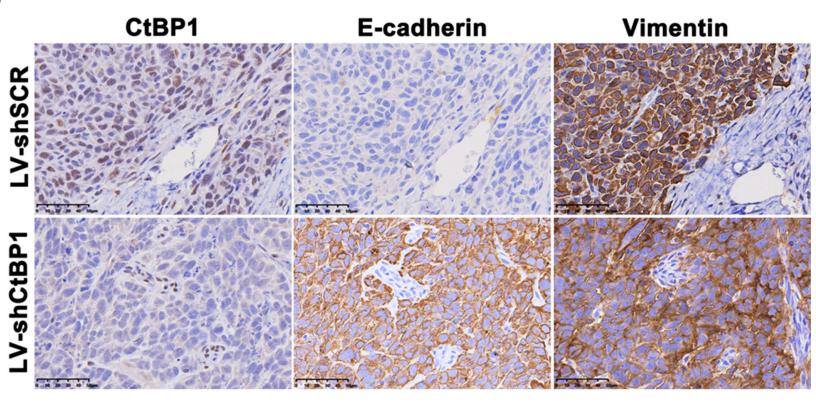

G

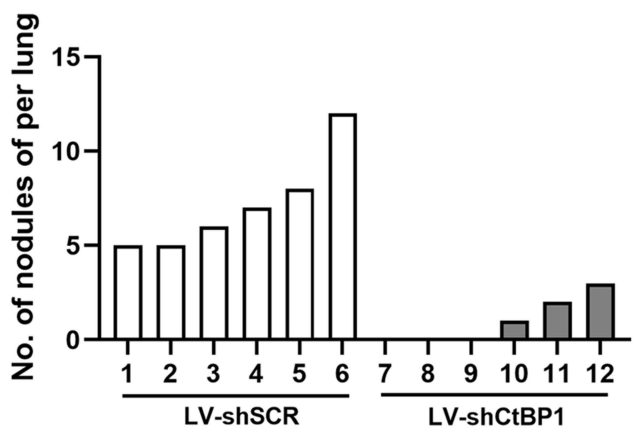

Figure 4 Knockdown of CtBPI reverses hypoxia-induced sarcomatoid transformation in HepG2 xenografts. (A) Knockdown of CtBPI inhibited the tumor growth of HepG2 xenografts. (B) H \& E staining shows that the control xenografts present more necrotic areas (N), less epithelial areas (E) and emerging sarcomatoid areas (S), while CtBPI knockdown xenografts show less necrotic areas and no sarcomatoid areas. (C) Immunostained pancytokeratin (CK) shows that most of the control xenografts lost the epithelioid phenotype, while the CtBPI knockdown xenografts maintained the epithelial marker CK except for the fibrous septa. (D) In CtBPI-silenced xenografts, the expression of the epithelial marker E-cadherin was upregulated and the expression of the mesenchymal marker vimentin was downregulated compared with those of the control group. (E) In vivo metastasis assays show that CtBPI knockdown subcapsular xenografts in the liver present fewer secondary lung tumorlets. $\mathrm{H} \& \mathrm{E}$ staining $(\mathbf{F})$ and numbers $(\mathbf{G})$ of secondary lung tumorlets also suggested a decreased metastatic potential with CtBPI knockdown. 
epithelial marker CK in the control group but the expression of this marker remained in almost all tumor tissues except for fibrous tissue separating tumorlets in the CtBP1 knockdown group (Figure 4C). Moreover, upregulated E-cadherin expression and downregulated vimentin expression were observed in the CtBP1 knockdown xenografts (Figure 4D). In addition, a significant reduction in the number of lung metastases in the CtBP1 knockdown group was observed (Figure 4E-G). Taken together, the data above indicated that CtBP1 mediated EMT and sarcomatoid transformation of HCC in HepG2 xenografts and that knockdown of CtBP1 reversed this effect.

\section{Discussion}

Sarcomatoid transformation or transdifferentiation has been reported in many kinds of carcinomas, especially unsuccessfully treated carcinoma. ${ }^{14,15}$ The mechanism of tumor cell reprogramming to initiate sarcomatoid transformation still needs to be determined. Genome-wide expression analysis revealed that dysregulation of epithelial-to-mesenchymal transition (EMT) drives the sarcomatoid transformation of bladder cancer, ${ }^{16}$ non-small-cell lung cancer (NSCLC) ${ }^{17}$ and cholangiocarcinoma. ${ }^{18}$ Hypoxia is believed to be an inducer of EMT in cancer. ${ }^{19}$ However, data on the role of hypoxia in the sarcomatoid transformation of cancers are lacking.

sHCC more often occurs secondary to primary $\mathrm{cHCC}$ in individuals that received transarterial chemoembolization (TACE), radiofrequency ablation (RFA), and percutaneous ethanol injection. Yamada et al reported that overexpression of hypoxia inducible factor-1 was linked with aggressive local recurrence of hepatocellular carcinoma after radiofrequency ablation therapy. ${ }^{20}$ Unfortunately, the oxygen load of the tissue microenvironment was unable to be detected. Hypoxia is a driver of EMT in cancer cells. ${ }^{19} \mathrm{CtBP} 1$, a transcriptional repressor targeting diverse transcriptional regulators, was identified as an oxygen sensor that mediates hypoxia-induced tumor cell migration. ${ }^{10,21}$ Previously, we reported that CtBP1 induced an EMT phenotype in HCC cells by downregulating the expression of its target gene E-cadherin. ${ }^{11}$ Taken together, these results suggested that CtBP1 mediates hypoxia-induced EMT in cancer cells.

In this study, we found that CtBP1 protein expression was upregulated in the sarcomatoid elements of sHCC compared with the carcinoma elements (also the cHCC area) by immunohistochemistry. Moreover, the sarcomatoid elements underwent EMT and showed an increased mesenchymal phenotype with downregulated E-cadherin expression and upregulated vimentin expression. These data identified a role of CtBP1 in sarcomatoid transformation of HCC. In addition, HepG2 cells stimulated with hypoxia showed upregulated CtBP1 expression and an increased mesenchymal phenotype with spindle-shaped morphology and an increased migratory ability. To verify the role of CtBP1 in hypoxiainduced sarcomatoid changes in HCC cells, we knocked down CtBP1 by lentivirus-expressed shRNA in HepG2 cells. Downregulation of CtBP1 expression partially reversed hypoxia-induced EMT and mesenchymal-like cell morphological changes. These in vitro data indicated that CtBP1 mediated hypoxia-induced EMT and sarcomatoid morphological changes in HCC cells.

Xenografts in nude mice showed that silencing CtBP1 attenuated the growth of tumors with less necrosis and decreased sarcomatoid elements. Moreover, sarcomatoid elements showed a sarcomatoid phenotype with a spindleshaped cell morphology. These in vivo data confirmed CtBP1's role in sarcomatoid transformation of HCC. Furthermore, the epithelioid elements adjacent to sarcomatoid elements showed higher HIF-1a expression than other epithelioid elements. This result suggested a hypoxic microenvironment near the necrotic area. Notably, sarcomatoid elements showed downregulated HIF-1a expression compared with epithelioid elements. Ku et al ${ }^{13}$ reported that HIF-1a was lost in the sarcomatoid elements of sarcomatoid renal cell carcinoma. Based on this finding, HIF-1a may be involved in the early stage of sarcomatoid transformation. However, the reprogramming mechanism of hypoxia-induced sarcomatoid transformation has not been fully elucidated.

In conclusion, CtBP1 played a key role in hypoxia-induced EMT and sarcomatoid transformation of HCC and could be a candidate target for the management of sHCC.

\section{Abbreviations}

HCC, hepatocellular carcinoma; sHCC, sarcomatoid hepatocellular carcinoma; cHCC, classic hepatocellular carcinoma; TACE, transarterial chemoembolization; RFA, radiofrequency ablation; CtBP1, C-terminal binding protein 1; EMT, epithelial-to-mesenchymal transition; DMEM, Dulbecco's modified Eagle's medium; PBS, phosphate-buffered saline; 
HE, hematoxylin-eosin; IHC, immunohistochemistry; IF, immunofluorescence; HIER, high temperature-induced epitope retrieval; DAB, 3,3'-diaminobenzidine; ECL, chemiluminescence.

\section{Acknowledgments}

This study was supported by the National Natural Science Foundation of China (Grant no. 81660485 to XLZ, Grant nos. 82060500, 81760491, and 81560441 to SJX) and the Natural Science Foundation of Guangxi Province of China (Grant no. 2020GXNSFAA159131 to XLZ, Grant no. 2020GXNSFAA159040 to SJX). Xiaoling Zhang and Xiaoyu Wang are co-first authors for this study.

\section{Disclosure}

The authors report no conflicts of interest in this work.

\section{References}

1. Shafizadeh N, Kakar S. Hepatocellular carcinoma: histologic subtypes. Surg Pathol Clin. 2013;6(2):367-384. doi:10.1016/j.path.2013.03.007

2. Liao SH, Su TH, Jeng YM, et al. Clinical manifestations and outcomes of patients with sarcomatoid hepatocellular carcinoma. Hepatology. 2019;69 (1):209-221. doi:10.1002/hep.30162

3. Wang JP, Yao ZG, Sun YW, et al. Clinicopathological characteristics and surgical outcomes of sarcomatoid hepatocellular carcinoma. World $J$ Gastroenterol. 2020;26(29):4327-4342. doi:10.3748/wjg.v26.i29.4327

4. Wu L, Tsilimigras DI, Farooq A, et al. Management and outcomes among patients with sarcomatoid hepatocellular carcinoma: a population-based analysis. Cancer. 2019;125(21):3767-3775. doi:10.1002/cncr.32396

5. Luo C, Xin H, Yin D, et al. Characterization of immune infiltration in sarcomatoid hepatocellular carcinoma. Aging (Albany NY). 2021;13 (11):15126-15138. doi:10.18632/aging.203076

6. Malouf GG, Flippot R, Dong Y, et al. Molecular characterization of sarcomatoid clear cell renal cell carcinoma unveils new candidate oncogenic drivers. Sci Rep. 2020;10(1):701. doi:10.1038/s41598-020-57534-5

7. Sung CO, Choi H, Lee KW, Kim SH. Sarcomatoid carcinoma represents a complete phenotype with various pathways of epithelial mesenchymal transition. J Clin Pathol. 2013;66(7):601-606. doi:10.1136/jclinpath-2012-201271

8. Conant JL, Peng Z, Evans MF, Naud S, Cooper K. Sarcomatoid renal cell carcinoma is an example of epithelial-mesenchymal transition. $J$ Clin Pathol. 2011;64(12):1088-1092. doi:10.1136/jclinpath-2011-200216

9. Tickoo SK, Alden D, Olgac S, et al. Immunohistochemical expression of hypoxia inducible factor-1alpha and its downstream molecules in sarcomatoid renal cell carcinoma. J Urol. 2007;177(4):1258-1263. doi:10.1016/j.juro.2006.11.100

10. Zhang Q, Wang SY, Nottke AC, Rocheleau JV, Piston DW, Goodman RH. Redox sensor CtBP mediates hypoxia-induced tumor cell migration. Proc Natl Acad Sci U S A. 2006;103(24):9029-9033. doi:10.1073/pnas.0603269103

11. Zhang XL, Huang CX, Zhang J, Inoue A, Zeng SE, Xiao SJ. CtBP1 is involved in epithelial-mesenchymal transition and is a potential therapeutic target for hepatocellular carcinoma. Oncol Rep. 2013;30(2):809-814. doi:10.3892/or.2013.2537

12. Vaupel P, Multhoff G. Fatal alliance of hypoxia-/HIF-1alpha-driven microenvironmental traits promoting cancer progression. Adv Exp Med Biol. 2020;1232:169-176. doi:10.1007/978-3-030-34461-0_21

13. Ku JH, Park YH, Myung JK, Moon KC, Kwak C, Kim HH. Expression of hypoxia inducible factor-1alpha and 2alpha in conventional renal cell carcinoma with or without sarcomatoid differentiation. Urol Oncol. 2011;29(6):731-737. doi:10.1016/j.urolonc.2009.08.007

14. Hsieh MS, Lin MW, Lee YH. Lung adenocarcinoma with sarcomatoid transformation after tyrosine kinase inhibitor treatment and chemotherapy. Lung Cancer. 2019;137:76-84. doi:10.1016/j.lungcan.2019.08.029

15. Li X, Wu D, Liu H, Chen J. Pulmonary sarcomatoid carcinoma: progress, treatment and expectations. Ther Adv Med Oncol. 2020;12:1758835920950207. doi:10.1177/1758835920950207

16. Guo CC, Majewski T, Zhang L, et al. Dysregulation of EMT drives the progression to clinically aggressive sarcomatoid bladder cancer. Cell Rep. 2019;27(6):1781-1793 e4. doi:10.1016/j.celrep.2019.04.048

17. Manzotti G, Torricelli F, Benedetta D, et al. An epithelial-to-mesenchymal transcriptional switch triggers evolution of pulmonary sarcomatoid carcinoma (PSC) and identifies dasatinib as new therapeutic option. Clin Cancer Res. 2019;25(7):2348-2360. doi:10.1158/1078-0432.CCR-182364

18. Seol MA, Chu IS, Lee MJ, et al. Genome-wide expression patterns associated with oncogenesis and sarcomatous transdifferentation of cholangiocarcinoma. BMC Cancer. 2011;11:78. doi:10.1186/1471-2407-11-78

19. Tam SY, Wu VWC, Law HKW. Hypoxia-induced epithelial-mesenchymal transition in cancers: HIF-1alpha and beyond. Front Oncol. $2020 ; 10: 486$. doi:10.3389/fonc.2020.00486

20. Yamada S, Utsunomiya T, Morine Y, et al. Expressions of hypoxia-inducible factor-1 and epithelial cell adhesion molecule are linked with aggressive local recurrence of hepatocellular carcinoma after radiofrequency ablation therapy. Ann Surg Oncol. 2014;21(Suppl 3):S436-S442. doi:10.1245/s10434-014-3575-z

21. Chen L, Yang Y, Xu L, Liu R, Wang Y. Integrated gene expression profiling and chromatin immunoprecipitation followed by sequencing: analysis of the C-terminal binding protein in breast cancer. J Obstet Gynaecol Res. 2017;43(9):1472-1480. doi:10.1111/jog.13400 


\section{Publish your work in this journal}

The Journal of Hepatocellular Carcinoma is an international, peer-reviewed, open access journal that offers a platform for the dissemination and study of clinical, translational and basic research findings in this rapidly developing field. Development in areas including, but not limited to, epidemiology, vaccination, hepatitis therapy, pathology and molecular tumor classification and prognostication are all considered for publication. The manuscript management system is completely online and includes a very quick and fair peer-review system, which is all easy to use. Visit http://www.dovepress.com/testimonials.php to read real quotes from published authors.

Submit your manuscript here: https://www.dovepress.com/journal-of-hepatocellular-carcinoma-journal 